\title{
A COMPARISON OF SOME MENTAL AND PHYSICAL TESTS IN THEIR APPLICATION TO EPILEPTIC AND TO NORMAL SUBJECTS.
}

\author{
BY W. G. SMITH, \\ Assistant Lecturer in Physiology, University of Liverpool.

\begin{abstract}
The aims of the investigation. The subjects examined in the course of the work: one normal group and two epileptic groups. The mental and physical tests and the methods employed. Results of the application of the tests. In recognition, immediate memory, and reactions there are in general marked differences between the groups. In sensory discrimination, rapidity of voluntary movement, rhythmic movement, and maximal voluntary contractions - processes which are relatively simple-the differences of the groups are slight. The effects of practice.
\end{abstract}

IT is the aim of the following paper to compare several experimental methods which may be used in investigating the condition of individuals suffering from mental disease, and to present the results which have been obtained by applying the methods to epileptic and to normal subjects 1 . One has to take into account in work with abnormal subjects not merely the intelligence and education, but also the likes and dislikes of the patient, and it is only by trial that one can discover what modifications of method are practically suitable. It was also intended when the work was planned to employ the methods in determining the nature of some of the changes which are brought about by the epileptic fit, and thus to contrast in individual patients the alternating phases of comparatively normal behaviour and behaviour as influenced by the fit.

1 The chief points in the investigation were brought before the Psychological Society, July 25, 1903. 
It was hoped in this way to avoid some of the difficulties which beset the comparison of a group of insane subjects with a group of normal subjects'. It was found in the end that the practical difficulties of securing an adequate number of observations made it impossible to carry out this project, and therefore the main body of results which are presented here deal with the relations between normal subjects and epileptic patients observed in the intervals between fits. One of these difficulties may be mentioned. A number of patients who had been brought over to the laboratory several times refused to come again, having formed the delusion that electricity was put into them by the apparatus employed in some of the experiments.

The work was carried on in the Pathological Laboratory of the London County Asylums, Claybury, Essex, during the years 1902 and 1903. I am greatly indebted to Dr F. W. Mott, Director of the Laboratory, for the opportunity of carrying on the work and for his constant assistance, and also to Dr R. Jones, Superintendent of the Asylum, for permission to examine the patients. During the investigation 23 male patients were examined, but it was finally decided to use the results from the 10 patients who aftorded the most numerous and reliable results. Two minor groups were formed, each including 5 individuals. In the first group the patients were comparatively rational in conversation and behaviour during the intervals between fits; in the second there was, on the whole, marked dementia. The average age was in the first group about 35 , in the second about 38 years. With these patients I have been able to compare a normal group of 6 subjects (men), about 26 years of age on the average. All, except one, were attendants in the asylum and may be regarded as belonging to the same social class as the patients. Observations were secured from the patients on 5 days, these days coming at irregular intervals. Five of the normal subjects contributed results on 3 days, one on 2 days. I regret that it was not possible to secure more numerous experiments in the normal group. It may, however, be assumed that a normal subject will tend to give more constant results, and that in this way the disparity in number of experiments between normal and epileptic groups may be of minor importance. Data will be presented later on by which some idea of the variations in the different groups may be gained. The tests which were applied dealt with the following subjects :-recognition; immediate

1 This principle has been already applied in investigating epileptic subjects by A. Gross, Verhalten einfacher psychischer Reactionen in epileptischen Verstimmungen, Psychologische Arbeiten, Leipzig, 1901, Bd. III. S. 385. 
memory; sensory discrimination; reactions involving movement and choice; rapidity of simple voluntary movement; tremor; rhythmic movement; maximal voluntary contractions. The object of these tests was to secure some indication not merely of sensory and ideational changes but of alterations in the processes of motor innervation.

The following brief notes, for many details of which I am indebted to Dr Pugh, Medical Officer in Claybury Asylum, give some particulars regarding the patients whose results are embodied in the tables. All the patients have fits of the grand mal type.

\section{Group I. Comparatively normal patients.}

A. Aged 20 ; gardener ; admitted Aug., 1900 ; duration of fits before admission 9 years; over 7 fits per month; quiet and rational in manner and talk during the times when he is in the laboratory; immediately after fits intractable and impulsive.

B. Aged 24 ; worker in piano factory ; adm. Jan., 1902; duration 3 years ; several severe fits since admission; emotional and very ready to talk; after fits excited and impulsive.

C. Aged 41 ; packing-case maker; adm. June, 1899 ; duration 8 years ; about 8 fits per month ; gives intelligent and graphic account of illness and history ; after fits violent and troublesome.

D. Aged 35; clerk; adm. Oct., 1893; duration 6 years; over 5 fits per month; intelligent, but rather simple; after fits violent and abusive and has auditory hallucinations.

E. Aged 56 ; waiter ; adm. Sept., 1900; duration of fits probably from boyhood; fits infrequent ; intelligent but moody ; after fits restless and developes auditory hallucinations.

\section{Group II. Patients showing marked dementia.}

F. Aged 31 ; shoemaker; adm. Sept., 1893; duration 7 years ; fits infrequent; dull and unintelligent; after fits impulsive and aggressive.

G. Aged 48 ; soldier; adm. Dec., 1897; duration 8 years ; fits frequent; talkative and rambling; after fits pugnacious and intractable.

H. Aged 47 ; belonged to band of travelling circus; adm. May, 1895 ; duration 10 years; about 8 fits per month; rambling and occasionally incoherent, with confused religious ideas; after fits electrical delusions.

I. Aged 44; messenger ; adm. March, 1895 ; duration 25 years ; fits frequent ; rambling and occasionally incoherent, very cheerful; after fits troublesome and pugnacious.

J. Aged 21 ; shoemaker ; adm. Sept., 1893 ; duration 2 years; about 5 fits per month ; simple and feeble minded; recovery from mental confusion after fits gradual. 


\section{Methods.}

Recognition. The experiments on recognition, with which the experiments were begun each day, were carried out by means of series of pictures and words. The pictures were reproductions of the paintings of great artists, which were printed on cards of the size of ordinary playing-cards: the words were typewritten on a slip of paper. A group of 7 pictures was selected and handed to the patient as soon as he bad seated himself at the table, with the request that he would look at them so as to be able to know them when they were shown again later on. After he had looked at the pictures as long as he pleased a series of 7 words relating to ordinary objects was given him and he was asked to read it aloud; he was again told to be ready to recognise the words later on. At the end of the hour the group of 7 pictures mixed up with 7 new ones was handed to him and he was asked to pick out the ones he had seen before. In the same way a slip of paper was given him bearing 7 new and 7 old words mixed up in 2 lines, and he was asked to say which words he had seen before. On one or two occasions when the words were inattentively or imperfectly read I asked the patient to read a second time, or when the recognition was not properly carried out I read the words aloud one by one, asking after each whether it had been seen before. By having an equal number of old and new pictures and words one can get a fairly precise idea of the nature and extent of the mental weakness. The patient may forget all the 7 pictures and words or part of them : he may completely confuse old and new and imagine he has seen all before, or the confusion may extend only to part of the pictures and words. Inasmuch as we have a predominantly visual process in the recognition of pictures while in the recognition of words we have probably a highly complex combination of visual, auditory, and motor processes we have the means of analysing in some detail the character of the changes in recognition.

Immediate memory. This process was tested by reading aloud to the subject a series of letters arranged at random which he was asked to reproduce the moment the reading was finished. The rate of reading was the same for all, and was regulated by a metronome beating at the rate of 108 per minute. The procedure was substantially the same as that employed in a former investigation on "The Range of Immediate Association and Memory"," the only difference being that whereas previously

1 Archives of Neurology, Vol. 1r. p. 767. This paper contains also some preliminary observations gained with the first test, viz., recognition. 


\section{Tests on Epileptic and Normal Subjects}

the series which were presented to the subjects contained 4, 5, 6 up to 10 letters, in this instance it was decided to omit the series containing 8 and 10 letters. The reason for this omission lay in the circumstance that as this test was only one of a number it was advisable to make it shorter and casier than on the former occasion when it was studied in detail. The series containing 4 letters (which we may call stage 4) was given first; the series containing 5 letters (stage 5) followed and so on. As a rule there were not more than three experiments at each stage on one day. By means of this test combined with that for recognition we can compare the memory for immediately preceding events with the memory for events occurring at a time further removed. As soon as these experiments were finished a pause was made to relieve the strain on the patient. He was encouraged to smoke and talk about the things that interested him, his health, his history, and so on.

Reactions. After this interval the reaction experiments were taken ${ }^{\mathrm{I}}$. A pack of playing-cards was divided into halves, one half being used for choice experiments, the other to determine the speed of the movements by which the cards were handled. In the choice experiments one card from each suit was laid in front of the patient and he was asked to distribute the rest of the cards into 4 heaps, each containing the cards of one suit. In the other set of experiments the patient was asked to throw the cards one by one on the table so as to form one heap. Two experiments were made each day with choice and with throwing: in both cases the time was taken by a stop-watch giving fifths of a second. The reason for using this method for reaction experiments lay in the wish to secure activities which would be more or less familiar to the subject and would possess some interest. The difficulty in that case is that it is hard to find an activity which is not strange or difficult and yet is not influenced by the previous habits of the individual. The disadvantages which arise from the fact that some persons are much more familiar with cards than others will be dealt with later on.

Sensory discrimination. The test for sensory discrimination, which was taken next, was carried out by means of lines drawn in black ink on sheets of paper, measuring 17 by $12.5 \mathrm{~cm}$. One sheet, with a line $100 \mathrm{~mm}$. in length, the standard line, was placed in front of the patient towards the left. On the right were placed in succession 15 lines

1 This method of studying reaction times by playing-cards was introduced by Jastrow, Science, Vol. virr. p. 237. It need hardly be remarked that the term 'choice' as used here is employed only for convenience and that more is involved than mere decision between alternatives. 
('compared lines') one of which was equal to the standard line while the others differed from it by $1,2,3,4,5,6,8 \mathrm{~mm}$., the number of lines that were longer than the standard line being equal to the number that were shorter. The patient was asked in each case to tell whether the compared line was equal to the standard line, or greater, or smaller than it. On the first few days the sheets were laid on the table in front of the subject; afterwards they were placed on a small stand which held them at an angle of about $30^{\circ}$ above the table. The observations made in these two ways have been taken together; the patients were allowed to look at the line in any way and for any length of time they pleased so that the difference of results due to the position of the lines may be regarded as negligible. The whole set of lines was presented twice each day; the order in which the lines came being determined by chance and being changed each time they were presented. It was suggested to the patients that they should regard this as a test for eyesight; they were in all cases encouraged by being told that they had done extremely well, and that a second trial might remove any small errors they had made.

Rapidity of movement, tremor and rhythm. The rapidity of voluntary movement was determined by finding the rate at which the patient could tap on a key which was connected with an electric signal writing on the recording surface; the problem before the patient was easy and simple, while the motive of seeing how quickly he could carry on the movement was one which might be expected to appeal to him. Owing to the fact that the Jacquet chronoscope was available for securing accurate records only in the later stages of the work, the results for tapping and for the following experiments on tremor are based on one day's work only. The rate of tremor was determined by getting the patient to press gently with the forefinger of his right hand on the button of a Marey cardiograph, which was connected with a delicate Chauveau tambour. The forearm was first rested on a box which brought hand and arm to the same level; after the observation had been continued in this way for about half-i-minute the box was removed and the tracing taken for some time longer. The object of this alteration was to separate in some measure the movements of the hand and of the arm. Rhythmic movement was tested by asking the patient to keep time to the beating of a metronome which was made to beat first at the rate of 60 beats per minute, then at double this rate. The patient kept time by pressing on a piece of rubber tubing lying before him on the table: one end of the tubing was 
closed, while the other was joined to the tambour. It was hoped to get indications not merely of the accuracy with which time was kept, but also of the character of movements made in carrying out the rhythm. The apparatus was so arranged that the patient sat in oue position and carried through the different movements without further trouble than that of moving to the table on which the apparatus was placed.

Maximal contractions. These observations on rapidity of movement, on tremor, and on rhythm were carried out together each day in the earlier stage of work. At a later stage this group of experiments was replaced by the work on maximal voluntary contractions. For this purpose a modification of Mosso's ergograph was used. In place of the weight attached by a string passing over a pulley to the moving carriage which bears the writing lever there was introduced a spring balance of such a strength that it could not be pulled out to its full extent. In place of the arm-rest with the attachment for the finger a piece of apparatus was arranged by which the force exerted in the grip of the hand could be registered. In this arrangement the hand grasped a fixed vertical pillar and a moveable vertical bar connected by a horizontal arm with the moving carriage: the grip of the hand bore directly in a straight line on the spring balance. There are several advantages in this form of ergograph. Allowing the recording surface to move at a moderately fast rate we get a tracing of the full extent of the contraction of the muscles of the hand. This was of importance, inasmuch as the aim was tot to secure a long series of contractions showing fatigue, but to get an analysis of a few maximal contractions. Further, one may expect in this way to secure the willing cooperation of the patient more readily than if he is asked to carry out a prolonged and fatiguing series of contractions. He was always asked to show how strong he was, the appeal being thus made to motives whose presence might be relied on. The signal for the patient to exert himself was the sound of an electric bell so arranged that when the bell was struck by the hammer another circuit passing through the hammer and the bell to the electric signal was closed and the moment of stimulation registered. The patient was requested to grip as hard as he could, and then let go as soon as he had done his best. 


\section{Results.}

In the majority of the tables which follow I have presented the results of the normal and the two epileptic groups in successive horizontal lines, thus facilitating comparison. It has not seemed advisable to lengthen the paper by presenting and discussing individual results and therefore the numerical data have been condensed as far as possible. At the same time the central values and the mean variations are presented in many cases in addition to the averages, so that a judgment can be formed as to the reliability of the averages. The central value is specially important in the treatment of short series of experiments, such as one has often to be content with in pathological work, for it gives us an objective means of minimising the effect of exceptional and disturbing values. It has, however, been used merely as a supplement, and the principal stress has been laid on the arithmetical mean or average, which represents better the mass of the results.

Recognition. In the following table the results for pictures and words appear separately. Under each of these headings are given two classes of error; $(a)$ those in which the objects originally presented are not recognised, the error being termed one of forgetting, $(b)$ those in which the new objects are thought to have been seen before, the error being one of confusion between old and new. In each case the average for each class of error has been first calculated for the individual; these individual averages form the basis of the general averages presented in the table. In the same way the mean variation (m.v.) for each error has been calculated first for the individual, and then, on the basis of this, for the group. The total number of experiments on which the general averages are based is 25 .

\section{TABLE I. Recognition.}

\begin{tabular}{|c|c|c|c|c|c|c|c|c|}
\hline \multirow[b]{3}{*}{ Groups } & \multicolumn{4}{|c|}{ Pictures } & \multicolumn{4}{|c|}{ Words } \\
\hline & \multicolumn{2}{|c|}{ Forgetting } & \multicolumn{2}{|c|}{ Confusion } & \multicolumn{2}{|c|}{ Forgetting } & \multicolumn{2}{|c|}{ Confusion } \\
\hline & $\begin{array}{l}\text { av. } \\
1 \cdot 2\end{array}$ & $\begin{array}{c}\text { m. v. } \\
0.5\end{array}$ & $\begin{array}{l}\text { av. } \\
0.8\end{array}$ & $\stackrel{\text { m. v. }}{0.4}$ & $\begin{array}{l}\text { av. } \\
1.7\end{array}$ & $\begin{array}{c}\overrightarrow{\mathrm{m} . \mathrm{v}} \text {. } \\
0.6\end{array}$ & $\begin{array}{l}\text { av. } \\
0.8\end{array}$ & $\begin{array}{c}\text { m. v. } \\
0.5\end{array}$ \\
\hline I. Epileptic & $1 \cdot 3$ & 0.7 & 0.7 & 0.5 & $2 \cdot 1$ & 0.8 & $1 \cdot 9$ & $1: 3$ \\
\hline II. Epileptic & $1 \cdot 7$ & $1 \cdot 0$ & $2 \cdot 5$ & $1 \cdot 2$ & $2 \cdot 2$ & $1 \cdot 2$ & $3 \cdot 3$ & $1 \cdot 3$ \\
\hline
\end{tabular}

In almost all the divisions of the table it is evident that there is much greater difficulty in recognising words than in recognising pictures. At the same time the relations between the groups are similar in both. From this we may conclude that the relations between 
normal and pathological groups are the same whether the processes in recognition are exercised on pictures or on words. And the fact that the relations are the same may be taken as evidence that the failure to recognise in the second epileptic group is not due to inability to read perfectly. With one exception there is an increase in the number of errors in each column as we pass from the normal to the first epileptic group and then to the second. It will be observed however that the increase is much more marked in confusion than in forgetting. In fact the preponderance of forgetting over confusion which holds in the normal and the first epileptic groups is changed to the opposite in the case of the second epileptic group. This fact has an interesting bearing on our experience of ordinary life. Everyone forgets many things from day to day, while errors of confusion occur relatively seldom and are much more noticeable when they do occur.

As it was not practicable to give more than one group of pictures and of words each day the mean variation in this test is of less value than in the other tests where more than one experiment was made on each day, and it is of course less in the normal group than in the epileptic groups. But taking the figures as our only means of comparing the groups in regard to constancy of results, we see that there is in every instance an increase of the mean variation as we pass from the first to the last group. If we take the central value for each individual, thus avoiding some quite exceptional results, and calculate the average of these values, we find that while the numbers differ slightly from those in Table I., being in every case somewhat lower, the relations of the groups remain the same in regard to each of the points mentioned above.

Immediate Memory. The first of the two tables which follow contains the results of the normal group, while the second contains the combined results of the two epileptic groups. The average number of correctly reproduced letters for all stages taken together was found to be nearly the same in the two epileptic groups, viz., $18 \cdot 7$ in the first and 18.0 in the second, while it was markedly different from the normal total 23.1. This being so it seemed best to treat the results of the two epileptic groups as being on the same level, and thus reduce the number of tables. For a detailed account of the way in which the valuation of the results is effected reference must be made to the paper already mentioned on "The Range of Immediate Association and Memory." It will be sufficient here to give the following brief explanation of the descriptive terms used in the tables. 
(1) Rightly placed denotes letters which are reproduced correctly in every respect: (2a) group transposed refers to letters in a group whose position has been changed, e.g., with a series $a b c d e f g$ we might have $a b c$ reproduced at the end ; (2b) inversion in right position refers to letters in a group which mutually change places while the group as a whole retains its right place ; $(2 c)$ inversion in wrong position refers to letters which are inverted in a group which does not retain its right place; e.g., with the above series we might have $b a$ reproduced at the middle of the series; (3) wrongly placed denotes letters which were in the original series, but retain no trace of their original position ; (4) omission refers to letters of the original series which are not reproduced; (5) insertion refers to reproduction of letters which were not in the original series; $(6)$ repetition refers to the repetition of letters already reproduced; ( 7 ) defect indicates the number of letters by which the reproduced series, whether right or wrong, on the average falls short of the correct number; (8) excess indicates the number by which the reproduced series exceeds the right number. At the head of each column is given in Roman numerals the number of letters presented at each stage. In all cases the values are obtained by taking the arithmetical mean of the total number of letters coming under each headiug. The number of experiments on which the individual averages are based differs slightly in the different subjects; in general the average for the group is based on 75 experiments.

\section{TABLE II. Immediate Memory: Normal Group.}

$\begin{array}{lccccc}\quad \text { Stage } & \text { IV } & \text { V } & \text { VI } & \text { VII } & \text { IX } \\ \text { 1. Rightly placed } & 3 \cdot 9 & 4 \cdot 8 & 4 \cdot 9 & 4 \cdot 9 & 4 \cdot 6 \\ \text { 2a. Group transposed } & - & - & - & 0 \cdot 2 & 0 \cdot 5 \\ \text { 2b. Inversion in right position } & 0 \cdot 1 & 0 \cdot 1 & 0 \cdot 3 & 0 \cdot 4 & 0 \cdot 3 \\ \text { 2 c. Inversion in wrong position } & - & - & 0 \cdot 1 & 0 \cdot 1 & 0 \cdot 2 \\ \text { 3. Wrongly placed } & - & - & 0 \cdot 1 & 0 \cdot 4 & 0 \cdot 8 \\ \text { 4. Omission } & - & 0 \cdot 1 & 0 \cdot 6 & 1 \cdot 0 & 2 \cdot 6 \\ \text { 5. Insertion } & - & 0 \cdot 1 & 0 \cdot 4 & 0 \cdot 6 & 0 \cdot 8 \\ \text { 6. Repetition } & - & - & 0 \cdot 1 & 0 \cdot 1 & 0 \cdot 4 \\ \text { 7. Defect } & - & - & 0 \cdot 1 & 0 \cdot 3 & 1 \cdot 4 \\ \text { 8. Excess } & - & - & 0 \cdot 1 & 0 \cdot 1 & 0 \cdot 1\end{array}$

TABLE III. Immediate Memory: Epileptic Groups.

1. Rightly placed

2a. Group transposed

2b. Inversion in right position

2c. Inversion in wrong position

3. Wrongly placed

4. Omission

5. Insertion

6. Repetition

7. Defect

8. Excess
IV

$3 \cdot 6$

$-$

$0 \cdot 1$

$-\overline{0 \cdot 1}$

$0.2 \quad 0.6$

$0.3 \quad 0.5$

$-\quad 0.1$

- $\quad 0 \cdot 1$

$0.1 \quad 0.1$

$\begin{array}{ll}\text { VI } & \text { VII } \\ 3 \cdot 8 & 3 \cdot 8 \\ 0 \cdot 2 & 0 \cdot 2 \\ 0 \cdot 4 & 0.2 \\ - & 0 \cdot 1 \\ 0 \cdot 3 & 0 \cdot 6 \\ 1 \cdot 3 & 2 \cdot 0 \\ 0.9 & 1 \cdot 2 \\ 0 \cdot 3 & 0.4 \\ 0.3 & 0.6 \\ 0.2 & 0.2\end{array}$

IX

$\mathbf{3} \cdot \mathbf{0}$

$0 \cdot 4$

$0 \cdot 3$

$0 \cdot 2$

$1 \cdot 3$

$3 \cdot 8$

$1 \cdot 6$

0.7

$1 \cdot 6$

0.1 
We have in this test fortunately a standard with which we can compare the results of these two tables. If we take the normal results obtained in the former research from 11 individuals, none of whom is included in the present group, and determine the number of rightly placed letters at all stages taken together, we find that the total is 23.2, a number practically identical with that of Table II, viz., 23'1. Again, the similar totals in the two groups which showed decided evidence of pathological weakening were $18 \cdot 4$ and $18 \cdot 3$, while the totals in the two epileptic groups are $18 \cdot 7$ and $18 \cdot 0$. In the light of these correspondences the similarity of the results in the two epileptic groups becomes less surprising: it means that both are markedly affected and touch a low pathological level. The lack of correspondence between this test and that of recognition in the case of the epileptic groups is evidently another expression of the fact which is so remarkable in individual cases, that immediate memory may be practically normal while memory for the ordinary experiences of each day is completely lost, and conversely ordinary memory may be retained while immediate memory is deeply affected. We may say in other words that the processes involved in the two tests do not run parallel, but are independent variables. When the central values for each stage in each individual's results are determined, and the averages taken, the figures for the groups in order are $24 \cdot 1,19 \cdot 5,19 \cdot 3$; i.e., the ratios are the same while the absolute values are somewhat higher.

When we examine the various classes of error in the two tables the pathological character of the epileptic results is confirmed. These results show a decided increase in the classes of wrongly placed letters, of omission, insertion, repetition, and a slighter increase in defect and excess : in other words, an increased associational disorder is shown by all these classes of error. On the other hand the classes $2 a, 2 b, 2 c$, which include all the letters that retain some trace of their former position, i.e., indicate partial disorder, are not markedly different in the two tables. This, again, confirms what was found in the former research that the evidence of pathological change is to be found not in errors indicating partial disorder but in those indicating relatively complete disorder.

That the difference between the epileptic groups though slight is not to be entirely neglected seems clear from the fact that it shows itself regularly in the various classes of error. The relations of the groups can be brought out most clearly by the following arrangement, in which the errors at all stages are added together: 


$\begin{array}{lcccccc} & \text { Wrongly placed } \cdot \text { Omission } & \text { Insertion } & \text { Repetition } & \text { Defect } & \text { Excess } \\ \text { Group I. } & 2 \cdot 1 & 7 \cdot 5 & 2 \cdot 8 & 1.0 & 3.9 & 0.3 \\ \text { Group II. } & 2.5 & 8.3 & 6.1 & 1.9 & 1.2 & 1.0\end{array}$

We see here that the increase of error is in the same direction at each point except one: the diminution of defect in the second group is accounted for by the increase in omission, insertion, and repetition.

We found in the first test that there was a marked increase of errors of confusion in the second epileptic group. The class of error in this test which corresponds to confusion is that of insertion. It is interesting to observe that the errors of insertion in the second group are more than double what they are in the first epileptic group: we have in this fact a valuable confirmation of some of the results in the first group. The anomalous result in the first test that errors of confusion in pictures were slightly fewer in the first epileptic than in the normal group is not supported by the present test: the errors of insertion at all stages in the normal group amount to 1.9 as against $2 \cdot 8$ in the first epileptic group?

A report on the patients' memory for time may be introduced here. During the latter part of the investigation they were asked how long it was since they had been in the laboratory before and how long their stay in the asylum had been. It is extremely difficult to estimate the value of the answers when they are sometimes given in vague statements such as "about a month" and sometimes are accurate to a day. In general, however, it seemed clear that the members of the first epileptic group had a better understanding and memory of time than those in the second group.

Sensory discrimination. There can be little doubt that the mental processes in sensory discrimination are simpler and demand less mental capacity than those involved in the preceding tests. On the ground that in mental disease it is the more complex and highly evolved activities which are most liable to suffer injury, we might expect that this test would show less difference between the three groups than do the others. This is in fact the result brought out in the following table.

Table IV. Sensory Discrimination.

$\begin{array}{lccccc}\quad \text { Groups } & \text { Correct judgments } & \text { m. v. } & \text { 'Longer' } & \text { 'Equal' } & \text { 'Shorter' } \\ \text { Normal } & \mathbf{9 \cdot 7} & 1 \cdot 3 & \mathbf{4 . 0} & \mathbf{5 . 6} & \mathbf{5 . 4} \\ \text { I. Epileptic } & \mathbf{9 \cdot 2} & \mathbf{1 . 3} & \mathbf{3 . 5} & \mathbf{6 \cdot 2} & \mathbf{5 \cdot 3} \\ \text { II. Epileptic } & \mathbf{1 0 \cdot 4} & 1 \cdot 2 & \mathbf{6 . 0} & \mathbf{4 \cdot 1} & \mathbf{4 . 9}\end{array}$

${ }^{1}$ It may be mentioned here that the hearing of the patients was tested and was found to be fairly good in all except one, who is included in the tirst epileptic group. 
The columns headed 'longer,' ' equal,' 'shorter' contain the average number of judgments of each kind made by the subjects. The proportion of correct judgments is seen at once when it is remembered that the possible total of such judgments was 15: of these 1 should have been 'equal,' while 7 should have been 'longer' and 7 'shorter.' As there were two judgments each day on each of the compared lines, the total number of experiments on which the general average is based is in each epileptic group 50.

The differences between the averages of correct judgments in the three groups are slight: if we lay any stress on them we must say that the second epileptic group is the more accurate, this being indicated not merely by the average but by the mean variation. This general result is confirmed by the fact that perhaps the most demented patient in the second group gave a higher average of correct judgments, viz., 12.9, than any other subject normal or abnormal, and that his mean variation was also the smallest in the three groups. The patient who gave the next highest value-11.4_was also very demented. We have in this test evidently a mental activity which is in no way affected by the mental enfeeblement that is evident in other respects. Here, again, we can employ the central value to discount the exceptional values; taking the average of the individual central values we find that the numbers do not differ appreciably from those based exclusively on the arithmetical mean, being for the successive groups $9 \cdot 7,9 \cdot 1,10 \cdot 5$. The variations in the averages of judgments in the last three columns do not appear to possess any significance.

Leaving aside the question of correlation with other tests we may attempt to estimate the factors which enter into the judgments. If we divide the subjects, normal and abnormal, into a higher and a lower group, one giving the larger number of correct judgments while the other gives the smaller, we find the relations to be those embodied in the following table.

\begin{tabular}{lccccc}
\multicolumn{5}{c}{ TABLE V. } \\
Group & Correct judgments & m. v. & 'Longer' & 'Equal' & 'Shorter \\
Higher & $\mathbf{1 1 . 5}$ & $\mathbf{1 . 1}$ & $\mathbf{5 \cdot 4}$ & $\mathbf{3 \cdot 4}$ & $\mathbf{6 \cdot 2}$ \\
Lower & $\mathbf{8 \cdot 0}$ & $\mathbf{1 . 5}$ & $\mathbf{3 \cdot 5}$ & $\mathbf{7 \cdot 2}$ & $\mathbf{4 \cdot 3}$
\end{tabular}

It is to be noted that the smaller mean variation goes with the more correct judgments : in other words, the more correct perceptions are also the more constant. In both groups there is a tendency to underestimation of the compared lines shown in the excess of judgments 
'shorter.' The factor which differentiates the groups in this relation is the greatly increased number of judgments 'equal' in the lower group; i.e. the failure is due not to a positive tendency to error in one direction or the other, but to inability to discriminate.

It might be supposed that the results of these tables would be distinctly modified by imperfect vision on the part of the subject. In view of this possibility all the subjects were examined with Snellen's test types. Two patients were found whose vision was unusually good: their correct judgments numbered 11.3 and 8.4 ; two were found with very defective vision; their averages of correct judgments were 10.8 and 9.6. In view of these facts it seems clear that the character of the results is not due in any appreciable degree to peculiarities of vision. It is quite conceivable that an individual whose vision is poor may on that account unconsciously take greater care in discriminating what is before him and so compensate for his defect.

Reactions. In the following table we have the time in seconds which is taken by the three groups, (a) to distribute cards into four heaps according to their suit, $(b)$ to throw the same number of cards in succession on one heap.

\section{TABl.E VI. Reactions.}

\begin{tabular}{|c|c|c|c|c|}
\hline \multirow{3}{*}{$\begin{array}{l}\text { Groups } \\
\text { Normal }\end{array}$} & \multicolumn{2}{|c|}{ Choice } & \multicolumn{2}{|c|}{ Throwing } \\
\hline & av. & m. v. & av. & $\mathbf{m}$ \\
\hline & $17 \cdot 0$ & $1 \cdot 6$ & $8 \cdot 3$ & 0.6 \\
\hline I. Epileptic & $23 \cdot 6$ & $2 \cdot 7$ & $11 \cdot 0$ & 1.0 \\
\hline II. Epileptic & $32 \cdot 0$ & $\mathbf{3} \cdot 0$ & $13 \cdot 6$ & $1 \cdot 2$ \\
\hline
\end{tabular}

It will be observed that there is no exception to the rule that as we pass from the normal to the first and then to the second epileptic group the length of time required and the mean variation distinctly increase. While the difference between choice and throwing times, which may be called pure choice time, is 8.7 in the normal group, it is 12.6 in the first and 18.4 in the second epileptic group. That these values are not unduly modified by exceptionally long reaction times may be concluded from the fact that the averages of the central values for choice and throwing are practically the same as the averages of the arithmetical means, being in the three groups respectively $16 \cdot 8,23 \cdot 7,32.0$ for choice, and $8 \cdot 2,10 \cdot 7,13 \cdot 4$ for throwing.

Before we can draw conclusions from these figures we have to inquire how far the results are influenced by the previous habits of the 
subjects. All the normal subjects have played some kind of card game. When we arrange the patients into two sets according to the speed with which the throwing was carried out we find that the three patients who said they had never played cards fell into the set which takes the longer times. This fact indicates that previous habits have influenced facility of movement. On the other hand, when they are arranged according to pure choice times, two of these three are in the quicker set, and one is almost exactly intermediate in value between the two sets. This would indicate that previous familiarity with cards was of little importance in regard to choice. As a fact two out of these three patients are included in the first epileptic group so that the quicker reaction in this group can hardly be due to greater familiarity with cards. We can finally disregard the results of these three patients and combine the rest of the values for the epileptics into one set of averages. When this is done it is found that the average choice time is $28.5 \mathrm{sec}$., and the throwing time is $11.5 \mathrm{sec}$., while the difference is $17 \cdot 0$, i.e. about double what it is in the normal group. It must be remembered that these reaction tests were arranged specially with the view of comparing the condition of the patients before and after fits. But even on the less satisfactory basis of a comparison of groups we have good ground for putting down the greater part of the retardation in choice and in throwing to the same causes which produce the differences in the other tables.

In the course of the choice experiments errors were sometimes made by the subjects, the cards being put on the wrong heaps. Sometimes the error was unnoticed: sometimes it was observed and corrected. In order to determine whether the errors unduly influenced the averages I have worked out revised figures for the experiments which included no errors. The revised values for the three successive groups are $16 \cdot 1,22 \cdot 2,31 \cdot 5$. It is clear from this fact that no substantial error was introduced by this factor into the averages of the table. There is no marked difference between the groups in regard to the number of errors. That the numbers are slightly greater in the normal group may be due to hurry and carelessness'.

1 In taking the central values into consideration above $I$ have given the averages of all the individual central values. We can however carry out the central values more thoroughly by rejecting the extreme individual values and taking the average of the remaining values -3 in the epileptic groups and 2 in the normal group. When this is done the following relations are brought out. In recognition (taking errors of forgetting and confusion together) the errors for the three groups in order are for pictures $\mathbf{1 . 5}$, $1 \cdot 7,3 \cdot 7$, and for words $2 \cdot 0,3 \cdot 0,4 \cdot 0$. In immediate memory the values of rightly placed 
Rapidity of movement and tremor. We have in the repetition of an elementary muscular activity, such as is involved in tapping a key, a test which, like visual discrimination, requires a relatively simple psychophysical activity. We might, therefore, expect that it also would bring out relatively slight differences between the groups. And when we look at the results we find that there is practically no difference between the groups. The rate of tapping in the first 8 seconds is 6.3 per sec. in the normal group and 6.2 in the first and second epileptic groups: the rate in the second 8 seconds is 5.9 in the normal group and 6.3 in the two epileptic groups. These values are of interest when we compare them with the averages for throwing cards: the form of movement involved in placing cards, as we saw, brought out distinct differences in the three groups. The conclusion seems to be justified that both in the motor and the sensory spheres it is the more complex processes which show themselves to be most affected. Compared with tapping, the coordinated contractions of the muscles in both hands required in holding and in placing the cards form a much more complex process'. In regard to nearly all the graphic records of tapping, normal and abnormal, one is struck by the regularity in the rate of succession of the movements. Occasionally there is a straight line traced by the electric signal where two or three indications of movement should have been found, such gaps being found both in the normal and the abnormal records.

The chief interest of the results which I am able to present in regard to tremor lies in their correlation with the data for voluntary movement. It has been asserted by Levy-Dorn ${ }^{2}$ on the basis of observations on normal individuals and on persons suffering from nervous disease that there is in general a close agreement in the number of voluntary contractions per second and the rate of tremor. He has suggested also that where the tremor rate of a patient could not be determined it could be inferred from the rate of voluntary movement. In the following table the epileptic subjects have been divided into two sets, according to the speed with which the voluntary

letters are $24 \cdot 0,19 \cdot 5,19 \cdot 7$. In sensory discrimination the correct judgments number $10 \cdot 5,8 \cdot 8,10 \cdot 7$. In choice the figures are $15 \cdot 7,24 \cdot 0,29 \cdot 8$, in throwing they are $7 \cdot 3,10 \cdot 8$, 13.0. It will be observed that there are only slight differences between these values and the averages already given and that in general the relations remain the same.

1 Cf. Jack, "On the analysis of voluntary muscular movements," Proc. Roy. Soc. 1895, Vol. LVII. p. 477.

2 Neurologisches Centralblatt, 1898, S. 522. A communication in regard to the relations of voluntary movement and tremor was made to the Physiological Society, Jan. 17, 1903.

J. of Psych. I 
movements are carried out, the number of patients in each group being six in this instance.

\section{TABLE VII.}

$\begin{array}{lccc} & \text { First 8 sec. } & \text { Tremor } \\ \text { More rapid } & 7 \cdot 0 & 7 \cdot 1 & 15 \text { sec. } \\ \text { Less rapid } & 4 \cdot 9 & 5 \cdot 4 & 9 \cdot 5 \\ & & \text { Second } 8 \text { sec. } & 8 \cdot 5\end{array}$

It is clear that if there were such a correspondence as has been maintained it ought to show itself not merely in the results for all the patients taken together, but for the two sets into which they have divided. The average rate of tapping for these 12 patients is 6.2 per sec.: the rate of tremor is 8.95 per sec. The same want of correspondence is shown in the detailed treatment of the table given above. So far as epileptics are concerned there is no ground for assuming an identity in the rates of the two processes. It is, however, clear from the table that, while there is no identity, there is a certain correlation of rates in so far as there corresponds to the less rapid rate of movement a less rapid rate of tremor. In other words, the rate of discharge of impulses to voluntary movement though less than that of involuntary movement of tremor is yet related to it in such a way that to the more rapid rate of voluntary discharge there corresponds a more rapid rate of involuntary discharge. The correlation, however, is not universal: there are three patients out of the twelve who do not follow this rule. There is a further fact which becomes obvious on considering the individual data,-that mental ability is in no way correlated with speed of voluntary movement: three patients out of the four who have the highest rates are included in the group of more demented epileptics.

It will be remembered that the observations on tremor were made first with the arm supported and then with the arm free. The data given above refer only to the tracings taken when the arm was supported. The tracings taken when the arm was free, instead of being clearer, were in nearly all cases much less distinct and much more difficult to read. The numbers for the more and the less rapid groups in Table VII are 8.1 and $7 \cdot 5$ when the arm is free, but I do not wish to lay stress on these values as the lesser numbers may in some cases be due to the difficulty of reading the curves even when the tracing was magnified. The observations do not give grounds for deciding 
how far the lessened distinctness is due to the character of arm tremor, and how far it is due to interference and summation of arm and hand movements.

Rhythm and maximal contractions. The observations in regard to rhythmic movement follow the rule of the simple voluntary movement in showing no appreciable difference between the two groups of epileptic patients with whom alone the experiments were made. There are differences in the way in which the pressure on the rubber tubing is made, some giving a slight pressure, others striking the tubing with some force at each beat. But with the exception of two in the second group all the patients were able to follow both rates of the metronome with approximate regularity. A more complex rhythm might have brought out differences, but would probably have made too great a demand on the intelligence of the patients.

The differences in the way in which various subjects carry out a maximal voluntary contraction are marked. Neglecting the 'latent period' between stimulus and rise of the curve we find differences in the rise of the curve, in the apex or plateau, and to a less extent in the fall. And the form of the curve is a constant characteristic which persists through the series of 10 or 12 contractions recorded each day and reappears on successive days in most of the cases with little change. The following tracings of two successive curves from three of the patients will serve as illustrations of the way in which the effort is carried out. The lowest line gives the time when the signal for the effort was given: the line above that gives the time in fifths of a second: the curves are to be read from right to left.
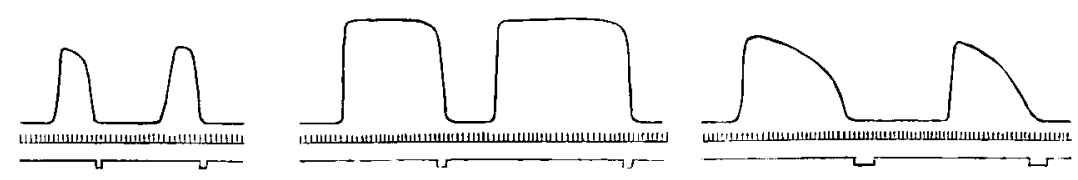

So far as can be seen the records cannot with certainty be correlated with the features of the epileptic condition. It may however be mentioned that there is in the curves obtained from some epileptics after the fit an alteration which consists in a gradual and prolonged rise of the curve. Some patients seemed unable, however often the matter was explained, to let go when they had given their maximal contraction, and kept up their grip till told to let go; but the observations are not sufficiently numerous to allow 
of general statements. The various types of curve are found both in normal and epileptic subjects. Perhaps we have here, as in former tests, a comparatively simple form of action in which individual differences are the prominent feature and are relatively unaffected by the general epileptic condition. Further analysis of single voluntary contractions, which I hope to undertake, and correlation with other forms and conditions of action may bring out the significant features, and show how the curves are modified by the force and persistence of the central innervation, the correlation of antagonistic muscles and the peripheral sense of effort.

The fact that there is a certain amount of correlation among the results of certain of the tests which have been employed, while in others the correlation fails, is in line with the conclusions of other investigators. It will be sufficient to refer to the work of Wissler? dealing with the varied tests which have been applied to students of Columbia University. At the conclusion of his investigation he makes the statement that "in summing up the foregoing it appears that all the tests in this series have little interdependence." Wissler, however, does not regard this result as proving the uselessness of such tests, but urges that they should be carried still further, in the belief that "because of the problems raised and the suggestions offered for new hypotheses, the making of tests is more desirable than ever." This point of view appears to have ample justification, both from the scientific and the practical aspects. It is only by carrying on such investigations that we can hope to escape from the vagueness and uncertainty of our views regarding the factors that determine mental ability and the various types of character. Negative results have perhaps more value in such investigations than they have elsewhere, inasmuch as they force on our attention the richness and complexity of mental life.

Practice. Under this heading I wish to bring together the facts which have been noted regarding the way in which the results varied in successive experiments on one day and on different days. For this purpose the results of the two epileptic groups taken together have been analysed for the first three days in order to show what changes took place, and to afford a comparison with the normal results which cover only three days. In general it may be said that the effects of practice, as shown in the results of successive days, and of 'Anregung'

1 "The Correlation of Mental and Physical Tests," Psychalogical Review, Monograph Supplements, Vol, III. No. 6. 
as shown in the successive results of each day, though present, are of minor importance.

In the test for recognition there is a distinct and fairly regular improvement in regard to both factors, forgetting and confusion, as we pass from the first to the third day. The following table in which the two classes of errors are combined shows the daily changes.

\section{TABLE VIII.}

\begin{tabular}{lcc} 
& \multicolumn{2}{c}{ First Day } \\
Subjects & Pictures & Words \\
Normal & $2 \cdot 8$ & $2 \cdot 4$ \\
Epileptic & 4.4 & 5.6
\end{tabular}
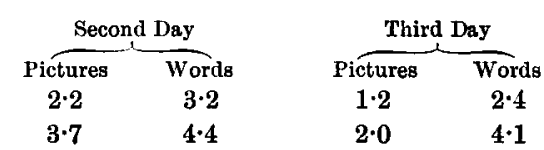

As the improvement is clearly visible on the third day both in normai and abnormal subjects we have evidently something more than increasing familiarity with what they were expected to do, and may fairly consider the decrease of errors as being largely due to practice. It seems fairly certain that if the normal subjects had continued their work as long as the patients the differences between normal and pathological groups would have been still more marked than they are. In this test we are, of course, unable to see the effect of successive experiments on each day.

In the test for immediate memory, on the other hand, we find that there is practically no improvement as we pass from the first to the third day of the epileptic results, and that the average for the three days is very nearly the same as the final average. There is, however, an improvement as we pass from the first to the third of the experiments with epileptics on each day, the values of rightly-placed letters for the three observations being 19.7, 18.2, 17.3. Seeing that there is no improvement from day to day in the abnormal groups we can directly compare the results of the normal and abnormal groups.

In the results for choice the values of the patients for the three days are $29 \cdot 9,28 \cdot 5,26 \cdot 7$ : there is, therefore, a distinct tendency towards shortening the reaction time, the tendency being rather more decided in the second epileptic group. There is a similar improvement in the normal group, the corresponding values being $18 \cdot 5,16 \cdot 5,16 \cdot 6$. There appears in the average values for the patients a very slight deterioration in the second experiment of each day: this, however, is due to the results of the first day ; on the other days there is a small improvement in the second place. In the normal group the second experiment of each day takes on the average $1 \cdot 1 \mathrm{sec}$. less time than the first. 
In throwing cards there is as we pass from the first to the third day a gradual lengthening of the time in the case of the epileptics, which is due to the second group, while in the normal group there is a slight improvement. The second experiment on each day gives a slightly shorter time in both epileptic and normal groups.

In sensory discrimination we have the curious result that there is on the whole an increase of errors from day to day in the epileptic results, the figures being $104,10 \cdot 5,11 \cdot 6$, and that the errors are more numerous in the second experiment of each day, the values being 5.3 and 5.5. One might be inclined to suspect these results as being accidental, were it not that one finds the same tendencies in the normal group in which the errors on the three days are $4.7,5 \cdot 5,6 \cdot 1$, and in the first and second places each day are $5 \cdot 3$ and $5 \cdot 6$. It is rather difficult to give a reason for these peculiarities: it is, however, to be remembered that the results of this test have a character quite distinct from that of the other results, and we may perhaps correlate this anomalous feature in regard to practice with the other anomalous relations of the groups as shown by this test. 